\title{
Essais
}

Revue interdisciplinaire d'Humanités

$2 \mid 2012$

Aux marges de l'humain

\section{Création sous contrainte}

Élisabeth Magne

\section{(2) OpenEdition}

Journals

Édition électronique

URL : https://journals.openedition.org/essais/10503

DOI : 10.4000/essais. 10503

ISSN : 2276-0970

\section{Éditeur}

École doctorale Montaigne Humanités

\section{Édition imprimée}

Date de publication : 15 novembre 2012

Pagination : 77-91

ISBN : 978-2-86781-857-8

ISSN : 2417-4211

Référence électronique

Élisabeth Magne, "Création sous contrainte », Essais [En ligne], 2 | 2012, mis en ligne le 17 janvier 2022, consulté le 20 janvier 2022. URL : http://journals.openedition.org/essais/10503 ; DOI : https:// doi.org/10.4000/essais. 10503 
Élisabeth Magne, laboratoire Clare/Artes, elisabeth.magne@ubordeaux3.fr

\section{Résumé}

Trois artistes contemporains introduisent le sujet et livrent leur pratique en exemple de créations sous contraintes. Les analyses révèlent des traits typologiques récurrents liés à la temporalité, à l'unité identitaire, à une forme spirituelle d'ascèse consentie. À rebours d'une histoire de l'art ayant travaillé dans le sens d'une levée des entraves - religieuses, politiques, techniques -, certaines pratiques contemporaines cherchent volontairement à réduire le champ des possibles et remontent les murs de la contrainte comme une discipline paradoxalement libératoire.

\section{Mots-clés}

Art contemporain, création, contrainte.

Abstract

\section{Creation under constraints}

Three contemporary artists introduce the subject and deliver their practice as examples of creations under constraints. Analyses reveal recurring typological features such as temporality, global identity, spiritual form of granted asceticism. Going against an art history having worked in the direction of levying obstacles - religious, political and technical ones -, a few contemporary practices try voluntarily to reduce the field of possibilities and raise the walls of the constraint paradoxically as a discharching discipline.

\section{Keywords}

Contemporary art, creation, constraints. 


\section{Création sous contrainte}

\section{Élisabeth Magne}

\section{Introduire le sujet 1 - une règle du jeu immuable}

L'histoire fascinante de l'artiste polonais Roman Opalka commence toujours par la description rituelle du programme de travail qu'il s'est fixé en 1965 et que seule la mort a clôturé en 2011 : peindre la suite des nombres entiers en partant de 1, puis 2, puis 3, etc. jusqu'à emplir la surface d'une toile de $196 \mathrm{~cm}$ sur 135. Puis poursuivre sur une autre toile à partir du dernier nombre de la toile précédente. Et ne jamais remettre en cause cette tâche infinie, chaque jour prenant la suite du travail de la veille dans une cohérence absurde et inéluctable.

À ce protocole immuable, l'artiste ajoutait après chaque séance de peinture, un autoportrait photographique, chemise blanche, lumière neutre, visage impavide où seuls les rides, les cheveux qui blanchissent et les traits plus marqués donnent la mesure du pas du temps, invisible à l'œil nu mais bien présent dans l'écart des années et des nombres peints. Le même pinceau $n^{\circ} 0$ renouvelé à chaque nouvelle toile et l'enregistrement des chiffres prononcés à haute voix en polonais au fur et à mesure qu'ils étaient écrits complétaient l'organisation rituelle de ce grand ouvre dont la progression ascétique n'a finalement pris tout son sens qu'à l'échelle d'une vie, ou plutôt à l'échelle de sa mort.

Et comme si la contrainte était encore trop ouverte, l'artiste avait choisi en 1972 d'additionner à chaque changement de tableau $1 \%$ de blanc au fond noir. C'est ainsi que depuis 2008 il ne peignait plus qu'en blanc sur fond blanc. Le 6 août 2011, la mort a arrêté son pinceau sur 5607249, suspendant une règle du jeu à laquelle il n'avait jamais dérogé.

Pour mesurer la rigueur de cette pratique quotidienne, on égrène volontiers les comptes, les chiffres, les mesures, les dimensions ${ }^{1}$. On calcule le nombre de

1. En 2009, le FRAC Aquitaine a proposé à l'écrivaine Noëlle Renaude de produire un texte très librement autour d'une ouvre de la collection. Elle a choisi les travaux d'Opalka. L'objet édité est assez fascinant et finalement très éclairant sur la démarche conceptuelle de l'artiste: courtes fictions où interviennent chiffres, listes, horaires, etc. témoignent de la prégnance de ce jalonnement temporel et spatial dans nos propres vies. Noëlle Renaude, De tant en temps, Bordeaux, Frac Aquitaine - Éditions Mix, coll. « fiction à l'œuvre », 2010. 
nombres sur chaque toile, le nombre de toiles par année, le nombre d'années par vie, le temps qu'il faut au blanc pour rejoindre le noir. C'est un empilement de jalons minuscules destinés à rythmer l'incommensurable où l'arithmétique vient ici nous rassurer et tenter de nous laisser saisir ce qui se dérobe.

À l'instar du collectionneur de timbres ou de porte-clés sans cesse suspendu à la pièce manquante et dont l'ouvrage reste toujours par nature inachevé, la force d'Opalka réside dans ce consentement prononcé une fois pour toutes et dans cette acceptation de l'incomplétude posée comme la règle définitive du rapport au réel et à ses choix.

\section{Introduire le sujet $\mathbf{2}$ - une déclinaison du temps}

En 2005, dans une structure de bois, Patrick des Gachons entrepose et présente vingt-quatre tableaux de $180 \mathrm{~cm}$ sur $180^{2}$. Dans ce dispositif, seule la surface de la dernière toile est visible, un carré bleu sur fond blanc. Mais qu'est-ce qui figure sur les vingt et une autres? Pour le savoir, il faut remonter à l'origine de la règle posée.

En 1983, à 39 ans - la moitié d'une vie? -, l'artiste peint au centre d'une première toile un carré bleu occupant très exactement la moitié de la surface disponible. Puis tous les ans, il augmente de $1 \%$ la surface du carré bleu sur les œuvres qu'il produit. L'ensemble présenté en 2005 est donc la compilation de ces toiles annuelles où grandit le bleu, avec ici un point mathématique en horizon d'achèvement: le remplissage de la totalité de la surface fixé en 2032 si l'artiste est toujours vivant.

Qui ne connaît pas l'œuvre de Des Gachons ne verra là qu'un jeu strictement formel, à dimension conceptuelle et abstraite. Pourtant l'attachement du peintre aux influences méditerranéennes offre une lecture plus sensible qu'il n'y parait au premier abord. L'envahissement du bleu, dans une échelle rapportée aux proportions d'une vie, devient traduction spatiale du temps. Des années portées à la fois à la visibilité et à l'ensevelissement. Une vie noyée peu à peu par le bleu. Il a ainsi pris la décision de lier son travail au temps, adoptant l'inexorable progression pour en rendre compte.

La technique utilisée, peinture à l'huile (carré bleu cobalt) sur acrylique (fond blanc) sur toile tendue sur châssis aluminium, est d'une extrême lenteur: afin d'atteindre l'intensité souhaitée du bleu profond, ce sont des dizaines de couches qui viennent patiemment à main levée achever la magnificence de la couleur. Le séchage est long, il impose son rythme et ce ne sont pas plus de deux

2. Patrick Des Gachons, Bloc de temps, présenté au CAPC Bordeaux en 2005, 24 pièces de $180 \times 180$ datées de 1983 à 2006. Cf. Jeannette Zwingenberger, "Les quatre horizons de Patrick Des Gachons ", in L'Euvre en programme, catalogue de l'exposition, CAPC Musée d'art contemporain de Bordeaux, Éditions Fage, 2005. L'artiste a également reconstitué une Colonne de vie, série où il remonte en amont de 1983 à rebours du pourcentage initial. 
ou trois toiles qui sont produites chaque année dans le respect du pourcentage du millésime. L'aliénation du choix, faite une fois pour toutes, est posée comme une libération: non point un retrait nihiliste mais un rassemblement sur la force sensible du vivant. Un retrait hors de la séduction du monde et de son mouvement clinquant pour s'en tenir à la palpitation pure.

La règle rituelle engagée ne vaut que pour celui qui la pose et se l'impose. C'est ainsi qu'elle peut apparaître comme absurde ou enfantine à qui la contemple de l'extérieur. Mais les années empilées dans le respect du protocole légitiment la dimension artistique de cette radicalité. La durée rend justice à la forme choisie et l'éclaire. Il suffit d'être patient!

Pourtant, les aléas de l'existence viennent parfois bousculer ce bel ordonnancement du travail. Dans ces cas-là, il est toujours troublant de voir comment ces œuvres extrêmement protocolaires s'adaptent au chaos qui pourrait les mettre en péril. Car les forces entropiques questionnent la règle et cherchent la jurisprudence nouvelle en conformité avec l'éthique établie. Ainsi Opalka avait mis au point lors de ses déplacements des "Carnets de voyage » dont les nombres s'intercalaient entre deux toiles. Pratique néanmoins compliquée qu'il abandonna ensuite car elle supposait une toile finie avant de quitter l'atelier pour quelques jours ou quelques semaines.

Plus étonnante la réponse que Des Gachons adressa au CAPC quant à la restauration d'une de ses toiles en 2010 : le musée lui ayant demandé son aide pour la réparation d'une œuvre ancienne, l'artiste procéda finalement à une "réactualisation " de la toile ainsi mise à jour, c'est-à-dire repeinte selon la proportion de bleu pratiquée dans l'année de la reprise.

\section{Introduire le sujet 3 - une discipline libératoire}

Depuis le 20 octobre 1993, Véronique Aubouy fait lire $\grave{A}$ la recherche du temps perdu de Marcel Proust par des centaines de gens de tous âges et de tous horizons. Là aussi, l'affaire est simple et le cérémonial bien réglé. L'artiste procède méthodiquement, dans l'ordre de l'histoire et chacun lit d'une traite en plan fixe environ deux pages in extenso. Le choix des lecteurs, d'abord des proches et des familiers, s'est étendu au gré des rencontres, des voyages, des recommandations, des candidatures spontanées à l'échelle du monde francophone. La réalisatrice, fascinée par la rythmique de l'écrivain, avait depuis longtemps trouvé chez Proust une sorte de paradigme fictionnel de son propre travail filmique. C'est en cherchant une forme iconique à cette accointance qu'elle eut l'idée de cette lecture ininterrompue, seule manière pour elle de respecter le fleuve de mots tant de fois lu et repris. Ainsi a démarré l'aventure de Proust lu.

Chaque lecteur choisit son cadre, son lieu, son environnement, construisant ainsi à mille voix le portrait sociologique d'une époque: dans son étable au 
milieu des poules caquetantes, assis dans sa cuisine, sur un banc de gare, dans sa boutique, à la plage, dans une chambre. On entend Proust mais aussi le monde d'aujourd'hui, des accents différents, des lectures plus ou moins hésitantes, comme une interférence croisée entre le temps de l'écrivain et celui du lecteur.

Celui que je filme prête son visage et sa voix au narrateur qui n'en a pas. Un télescopage entre l'écrit et l'oral. Par le rythme de sa lecture, par les pauses quil marque, il dévoile un temps qui lui est propre. [...] Proust lu est une somme d'actions et de mémoire. Par la répétition ad vitam de ce geste (appuyer sur le bouton record, geste fait à ce jour 990 fois), se trouve mon temps à moi. Je décline cette action comme on respire, comme on mange. C'est plus une liberté qu'une utopie [...]. Encore trente ans pour en avoir fini. Je rêve du jour où je n'y arriverai pas...

Interrogée sur cette échéance inquiétante, l'artiste explique avoir cherché des stratégies de ralentissement ou avoir réquisitionné d'autres textes de Proust autour du grand œuvre. Mais sans s'en satisfaire vraiment. Car le protocole ne peut se déliter sans mettre en péril l'édifice lui-même. Il ne s'agit pas seulement de s'en tenir à une radicalité intransigeante pour le seul bénéfice d'une allégeance à un quelconque dieu de la contrainte. Mais de trouver un équilibre personnel dans cette réitération du même, finalement très confortable aux dires de Véronique Aubouy ${ }^{4}$. La réalisatrice affirme puiser une très grande stabilité dans ce projet étiré à l'échelle d'une vie, projet qui fabrique toujours de l'activité et de la vie sociale quel que soit le tempo des autres œuvres en cours. Finalement, cette quotidienneté rassurante s'est vite imposée comme un pivot de vie, quelque chose à quoi elle peut se référer en permanence comme un endroit qui ne pose pas question, une habitude familière qui fonde son identité. On comprend mieux l'inquiétude de cette échéance, encore lointaine mais qui se rapproche inexorablement. À la question de cette fin, elle répond simplement qu'elle recommencera sans doute au début, à la première page. Pour ne pas en sortir. Pour ne pas écrire le mot Fin.

À l'instar des deux exemples précédemment cités, la saisie du projet appelle encore et toujours des chiffres, des dates et de l'arithmétique. Le nombre des lecteurs, leurs noms, la durée du montage, le nombre de pages restantes, le point possible de l'achèvement, autant de données souvent présentées autour

3. Texte écrit par la réalisatrice en 2007, disponible sur son site www.veronique.aubouy. fr/proust-lu.html On pourra également se référer à l'article de Françoise Nicol, «Proust lu, un film vidéo de Véronique Aubouy ou le secrétariat du temps ", in Jean Cléder et Jean-Pierre Montier (dir.) Proust et les images, Presses Universitaires de Rennes, 2003.

4. Entretien personnel avec Véronique Aubouy, février 2012. 
de la projection du film comme des litanies fascinantes où le temps mis en scène s'étire spatialement en répertoires, listes, mots comme à La Force de l'art en 2009 sous les voûtes du Grand Palais.

\section{Histoire d'un paradoxe}

Rarement créations auront produit autant de commentaires en forme d'inventaires et de données chiffrées. Le travail de ces trois-là, on le voit bien, donne lieu à des descriptions où jamais n'est faite l'économie de l'énonciation de la règle, de son mécanisme, de sa rigueur, de sa permanence. Sa légitimité s’y énonce en termes quantifiés destinés à étayer sa simplicité fragile. Car ces protocoles ne font qu'étirer à des dimensions monumentales des pratiques très élémentaires, voire primitives: écrire une suite de nombres, élargir un carré bleu, lire quelques pages. Seule leur itération - leur rabâchage? - les fait basculer dans le faire artistique, car elles y deviennent démonstration d'une nécessité indéfectible et inéluctable, ne souffrant plus de retour en arrière. Pour Opalka, la loi est même totalitaire, couvrant l'ensemble de la production sans aucune pratique dérogatoire hors de son champ. Pour Patrick Des Gachons et Véronique Aubouy, elle est l'œuvre constante mais accepte néanmoins qu'il y ait des travaux mitoyens, des déclinaisons, des fabrications autres en parallèle.

Néanmoins, la détermination est de même qualité: la volonté programmatique accrochée une fois pour toutes à une forme dégage l'artiste de tout flottement ou de toute hésitation quant aux aléas de son travail. En renonçant volontairement à l'amplitude des possibles de la création, ceux-là se dérobent à l'emprise illusionniste du choix, échappent à la séduction des écoles, des mouvements, des influences, posent leur temps hors du temps.

De même, pour saisir l'insaisissable et rassurer l'économie de nos vies, tentons-nous des inventaires, des comptabilités, des collections, des carnets, des albums, de la mesure, des additions et des soustractions, des écritures du temps.

Souvent de manière plus légère. C'est sans doute la même interrogation pseudo-existentielle qui fait lister à Umberto Eco ce qu'il a bien pu faire pendant une année non bissextile de $8760 \mathrm{~h}$ une fois enlevé le temps du sommeil, de la toilette et des repas: environ $8104 \mathrm{~h}$ à travailler comme professeur, directeur de recherche, conférencier et directeur de revue. Ce qui lui laisse, dit-il, 1 h 49 par jour «qu'il a occupé comme suit: sexe, échange avec les amis et la famille, enterrements, cures médicales, shopping, sport, spectacles... ${ }^{5} »$ !

Les artistes précédemment évoqués constituent des figures extrêmes de l'arithmétique temporelle et de la création sous contrainte ramassée sous l'unicité rassurante d'une pratique répétitive. Toutefois, cette mise sous tutelle

5. Umberto Eco, Comment voyager avec un saumon, nouveaux pastiches et postiches, LGF Livre de poche, 2000. 
semble aller à rebours du sens d'une histoire de l'art souvent associée à la levée des restrictions liberticides. S'affranchir de l'emprise du maître, de l'Église, du commanditaire, du Salon comme autant de formes coercitives est un défi que les artistes n'ont cessé de relever. De l'artisan, contraint dans sa réalisation par la religion ou le commanditaire, à la figure singulière de l'artiste contemporain, les étapes semblent aller dans le sens d'une autonomie revendiquée, conquise et assumée.

En 1454, lorsqu'Enguerrand Carton peint le Couronnement de la Vierge pour l'église de Villeneuve-Lès-Avignon, ce ne sont pas moins de 29 articles rédigés devant notaire dans le "prix-fait " qui imposent personnages, hiérarchie et composition, usage des couleurs, iconographie, etc. Le peintre est un fabricant, habile certes, mais uniquement un fabricant, et son travail relève des arts mécaniques. Il faut donc qu'il en laisse la conception aux lettrés, aux religieux, aux docteurs de l'Église. À ceux qui peuvent payer ses compétences manuelles et maîtriser le sens des images qu'il produit.

"L'expression d'un créateur ne s'exerce que rarement hors des limites ou contraintes imposées mais leur dispositif est plus ou moins lourd selon les époques ", rappelle Maurice Fréchuret ${ }^{6}$. Car l'artiste est sous surveillance iconographique, technique, économique, politique et religieuse; surveillance dont il ne cessera de vouloir se dégager dès le début de la Renaissance ${ }^{7}$. On connaît la puissance de conviction des images et l'encadrement dont elles ont fait l'objet. Académies, pouvoir royal et religieux s'emploient à restreindre et orienter le regard du peintre sur des sujets maîtrisés. Peine perdue! Caravage peint de vieilles femmes, et Chardin fait de la nature morte un genre sublime.

Ce sont ainsi différentes formes d'émancipation qui jalonnent le devenir de ce travailleur très particulier. Citons ici brièvement celle de l'artiste romantique, apothéose de la figure du génie, habité et mu par son seul pathos, forcément famélique puisqu'indifférent à la première des contraintes, celle de la réalité économique. Cette position parfois chèrement payée signe une étape importante dans la souveraineté de l'artiste, producteur d'abord pour luimême, en attente d'une reconnaissance dont il attend qu'elle vienne jusqu'à lui comme un dû, eu égard à son génie! Iconographie et choix techniques lui appartiendront désormais: un radeau à la dérive, des gares, les prostituées de Barcelone. Tout peut faire figure et la ressemblance, in fine, n'a plus à se référer au réel.

Autre étape franchie vers l'autonomie, celle du Dadaïsme et des ready-made duchampiens où la légitimité du travail se fonde sur sa seule intentionnalité de

6. Maurice Fréchuret, La Machine à peindre, Éditions Jacqueline Chambon, 1994, p. 162.

7. On se référera ici à quelques ouvrages de Nathalie Heinich, Etre artiste, les transformations du statut des peintres et des sculpteurs, Klincksieck, 1996 et L'Élite artiste: excellence et singularité en régime démocratique, Paris, Gallimard, 2005. 
l'artiste, sur l'abolition de la forme remplacée ici par l'attitude de l'individu, attitude qui se suffit à elle-même et n'entend engendrer aucune fabrication véritable. Avec Duchamp se confirme la prééminence de l'idée, la cosa mentale de Léonard de Vinci portée à son terme ultime. L'attitude vaut également comme contestation du pouvoir édificateur, comme moyen de se défausser de toute responsabilité dans l'acte de création et vient ainsi déranger des formes d'implication dans le travail que l'on pensait immuables.

Les grandes ruptures du $\mathrm{Xx}^{\mathrm{e}}$ siècle actent ainsi un certain nombre de positions ouvrant l'immensité d'un champ de possibles désormais disponibles et recevables. Libéré d'un métier et de ses apprentissages s'il le souhaite, l'artiste choisit sa forme, sa technique, ses sujets, ses matériaux. Il peut d'ailleurs ne plus rien fabriquer, mais simplement rédiger un projet d'œuvre, écrire un concept et en déléguer la réalisation à d'autres. Il peut faire tatouer en Chine par des spécialistes des cochons qu'il revend à prix d'or aux musées. Il peut remplir une baignoire de graisse, dialoguer avec un coyote, se faire suivre par un détective, dormir chez les autres, manifester dans la rue, dessiner des cercles dans le paysage ou peindre comme Vermeer très lentement des portraits à peine flous. Il semble donc qu'il puisse tout faire y compris ne rien faire.

Aucune routine ne l'enchaîne a priori selon la lecture marxiste où la production artistique apparaîtrait comme la forme la plus aboutie du travail expressif favorisant l'épanouissement de l'individu; ce que devrait être, selon Marx, l'idéal du travail libre valorisant l'individu. Dans son Portrait de l'artiste en travailleur ${ }^{8}$, Pierre-Michel Menger va plus loin encore en posant les valeurs cardinales de la compétence artistique - l'imagination, le jeu, l'improvisation, l'atypie comportementale, voire l'anarchie créatrice - comme la forme la plus avancée des nouveaux modes de production et des nouvelles relations du monde du travail. L'artiste y devient la figure exemplaire du nouveau travailleur portant l'idéal d'une activité à forte valeur ajoutée comme les aiment les chasseurs de tête de l'innovation. Les parcours du chercheur comme de l'artiste, fortement individualisés, comme dégagés de toute contingence sont ici capables de produire les manifestations les plus improbables de l'invention. C'est le règne des inégalités légitimées, portées par cette dimension concurrentielle fortement valorisée. L'artiste exemplifie dans cet essai le paradigme de la liberté innovante, interrogeant sans cesse le cadre dans une forme free-lance du lien sans asservissement et sans attaches.

Toutefois, cette indépendance conquérante et victorieuse est en grande partie illusoire et soulève un problème connexe, celui de la proclamation de ces pratiques comme étant de l'art, de la déclaration de leur légitimité. Se greffe ici

8. Pierre-Michel Menger, Portrait de l'artiste en travailleur, Métamorphoses du capitalisme, Paris, Le Seuil, coll. «La République des Idées », 2002. 
un jeu très complexe de cercles de reconnaissance où se disent le champ et le hors-champ. On se référera ici à la lecture de Nathalie Heinich sur ce qu'elle appelle Le Triple Jeu de l'art contemporain. Sans la maîtrise ou la connaissance de cette régulation sophistiquée, nul ne peut espérer se glisser dans les cercles de légitimation de cette vaste machine à trier les pratiques. Car le constat de la diversité, de tout ce qui peut être acceptable en termes de production et d'attitudes est assez vertigineux. C'est d'ailleurs cette diversité-là qui rend l'art contemporain si suspect aux yeux des non initiés. Comment reconnaitre le vrai de l'art lorsqu'il ressemble autant à la banalité du vécu ou même qu'il ne ressemble à rien? Lorsque toutes les positions deviennent a priori recevables. Car le tournant du $\mathrm{XxI}^{\mathrm{e}}$ siècle se caractérise par son anti-dogmatisme, par une dilution du concept d'art; la pratique artistique repose désormais sur un scénario plus ou moins aléatoire qui en fait non plus un magistère, non plus une expérience, mais une avancée aléatoire dans le territoire protéiforme de la réalité.

Ce que Jean-François Lyotard a nommé la condition postmoderne où il constate la fin des grands récits, renvoie à cet émiettement de la forme, à ce flottement du sens qui peut venir se poser n'importe où par la seule volonté du faiseur d'art. À charge pour lui de faire nommer son travail comme étant légitime, via tout l'arsenal énonciatif dont nous parlions plus haut.

\section{Inventer son unité, énoncer le champ clos de son existence}

L'immensité du choix néanmoins peut sembler terrifiante. Elle ne dit rien du chemin à parcourir pour l'apprenti artiste, des étapes à franchir et elle fabrique une instabilité potentielle parfois mal vécue: suis-je bien à l'endroit qui me révèle? Quel acte poser dans cette indétermination? Faut-il poursuivre et persévérer ou bien changer d'orientation? Sans cesse, le métier peut être remis en cause et reconstruit selon d'autres formes. L'identité est à élaborer de toutes pièces sans régulation contextuelle intempestive et interventionniste si ce n'est la gestion de la posture et du discours qui nomme le processus.

A rebours de cette disponibilité parfois opportuniste, fuyant les sirènes des recherches sans cesse remises en question, certains artistes choisissent de remonter pour eux-mêmes les murs de la contrainte, de prendre appui sur une structuration protocolaire affirmée comme le terminus ad quem de leurs interrogations d'artistes. Radicalement. Les trois exemples proposés en introduction en constituent des formes extrêmes, fascinantes dans leur asservissement à un motif répétitif.

Revenant sur cette servilité volontaire, Maurice Fréchuret écrit ceci:

L'artiste contraint des périodes anciennes [...] se révèle toujours prompt à déjouer les règles ou, pour le moins, à composer avec elles, à

9. Jean-François Lyotard, La Condition postmoderne, rapport sur le savoir, Minuit, 1979. 
délimiter, à l'intérieur du dispositif, une sphère dans laquelle ouvrer personnellement. Un artiste contemporain, qui n'est contraint en apparence par aucune instance extérieure, nullement freiné dans ses aspirations par quoi que ce soit, se détermine pour un art dont, jamais, même pas dans le très sévère procès de fabrication des icônes, la manière de faire n'a été aussi drastiquement réglementée. ${ }^{10}$

Et il poursuit plus loin sur l'un de nos exemples:

Opalka tourne le dos aux artifices maniéristes et aux développements sans fin des recherches sur la forme ou la matière. Paradoxalement, la propension comptabilisatrice et accumulatrice [...] se révèle économe quand il s'agit de définir les moyens d'action: soustraire, délaisser, dépouiller, renoncer. ${ }^{11}$

Art pauvre, art de l'ascèse, art centripète tout entier contenu dans le programme, dans sa clôture; le vocabulaire n'est pas sans évoquer une réclusion spirituelle en retrait du monde et de ses séductions miroitantes.

Toutefois, à la manière d'un horizon asymptotique, pourquoi ne pas revisiter d'autres pratiques bien plus nombreuses qui tendent vers des formes restrictives comparables à nos trois exemples introductifs? Sur l'échelle de Richter des formes protocolaires, nous pourrions ainsi relever quelques exemples d'artistes s'entourant d'une certaine rigueur dans la mise sous tutelle des potentialités. Eux-mêmes parleraient d'abord de choix, de style, d'identité plasticienne. Mais lorsque ces choix ferment un grand nombre de portes, lorsqu'ils semblent restreindre par trop les alternatives, lorsqu'ils s'étirent à l'échelle d'une vie, lorsqu'ils lient intrinsèquement un nom à un matériau, à une forme, à un concept, alors ils peuvent à leur tour être appelés contraintes. Comment nommer ces restrictions partielles, pourtant durablement invasives? L'expression paradoxale de "contrainte légère" résonnerait ici comme un oxymore qui maîtriserait mal son sujet. Parlons plutôt de contraintes électives pour approcher quelques noms de l'art contemporain à la lumière d'une telle grille de lecture.

En termes de décisions maintenues ouvertes ou partiellement fermées.

De tels renoncements pourraient même donner lieu à un essai de typologie selon les modalités de mise en œuvre de ces contraintes électives, typologie qui nous permettrait d'examiner les enjeux liés à chaque stratégie.

Un premier critère de classification pourrait rassembler ceux qui ont choisi de réduire drastiquement la matérialité de leurs propositions à une seule composante.

10. Maurice Fréchuret, La Machine à peindre, op. cit., p. 163.

11. Ibid. 
On reconnaîtra ici le noir de Soulages et ses jeux avec la lumière à l'exclusion depuis des décennies de toute autre ouverture colorée. La rigueur de cet ostracisme étendue à l'échelle d'une vie renvoie là aussi à un discours d'ascèse, de spiritualité dans les propos qui entourent cet artiste. Les commanditaires ne s'y sont pas trompés qui lui ont notamment réclamé des vitraux d'église, signant ainsi à Sainte Foy de Conques la rencontre d'une discipline avec l'économie esthétique d'une église de pèlerinage. Étonnamment, Pierre Soulages parlant de sa pratique d'artiste semble avoir fait ce choix comme pour mieux accueillir l'imprévisibilité de l'acte créatif:

L'artisan, le très grand artisan, sait ce qu'il va faire et comment il va sy prendre. Il connaît la fin de son activité et les bonnes méthodes à employer. Nous, nous ne savons pas ce que nous allons faire, ni ce qui va se faire indépendamment de nous. ${ }^{12}$

Citons encore les bandes verticales alternées blanches et colorées de $8,7 \mathrm{~cm}$ de Daniel Buren répétant à l'infini et sur tout support ce motif fabriqué industriellement pour des toiles de stores. Buren lui-même revendiquait le caractère impersonnel de ce motif, sa banalité quotidienne, sa présence sur de nombreux objets qui nous entourent. Pourtant et dans le même mouvement, il rappelait qu'une telle largeur constitue la distance moyenne de l'espacement entre deux yeux, loin du pathos de l'artiste et pourtant fonctionnellement rapporté à son corps. On trouve dans les écrits proposés par Buren à la fois cette interpellation mécanique - prendre un tissu ready-made - et ce lien à la corporéité que la rayure a toujours entretenu ${ }^{13}$. Pourtant en s'en tenant à un motif unique, Buren évacue la question de l'intention pour se concentrer sur le « comment? » du rapport à l'espace. En éliminant des possibles, il peut faire jouer - au sens où jouent une mécanique ou une pièce de bois -, l'adaptabilité du motif et plier cette rhétorique aux exigences de nouveaux lieux.

Autre adepte du in situ, Felice Varini, certes moins radical, a néanmoins réduit depuis longtemps ses figures de peinture à la ligne, au cercle, au carré et au triangle. Des chalets suisses aux chantiers de Nantes, toute son œuvre est une déclinaison permanente de ces quelques figures géométriques, réduites à des aplats colorés.

Que retenir de cette courte liste qui pourrait s'étendre à bien d'autres noms? Aucun d'eux ne parle de cette rétention formelle comme d'une contrainte mais bien plutôt comme d'un engramme évident à partir duquel peut s'ouvrir le travail, d'un préalable permettant d'aborder la diversité du réel avec une

12. Bruno Péquignot, "L'imprévisible et l'incertain ", in Dominique Berthet (sous la dir. de), L'Imprévisible dans l'art, L'Harmattan, coll. " ouverture philosophique », 2012, p. 170.

13. Cf. ici Michel Pastoureau, L'Étoffe du diable, une histoire des rayures et des tissus rayés, Poche, coll. «Points », 2007. 
identité posée, de ne pas se perdre dans les propositions mais bien au contraire, d'adapter sa propre matière à la mouvance du monde, de faire émerger le point de rencontre. Les productions de ces trois-là, aisément identifiables visuellement par les spectateurs que nous sommes, en deviennent d'ailleurs transparentes: ne reconnaît-on pas de loin un Buren ou un Soulages? La composante formelle retenue par chacun d'eux se fait substitut identitaire parfaitement identifiable et règle une fois pour toutes la question du pourquoi. Le sens de l'œuvre n'intervient pas ou plus. Ne restent finalement que les interrogations sur la fabrication. Demande-t-on à Varini pourquoi il fait des triangles? Guère. Mais les questions se bousculent sur le «comment » de la fabrication, sur la matérialité de son travail, sur l'échelle, sur les modalités de raccord entre sa peinture et les toits des hangars. Paul Ardenne dans Art, le présent y voit une symbolique de l'activité, une valorisation volontariste de la pratique $^{14}$. Le regard se déporte vers les conditions d'ajustement d'une forme élue - dont on ne parle plus - en regard d'une instabilité locale. Inaccessible, la contrainte originelle est une sorte de tache aveugle, hors de portée du faire car trop simple, trop radicale, inscrite comme une donnée séminale préalable à la mise en ouvre.

Autre critère fonctionnel possible pour cataloguer nos contraintes électives, la délégation de création: sous les étiquettes d'art contextuel ou d'esthétique relationnelle, beaucoup d'artistes actuels choisissent de se démettre de leur responsabilité de décideurs du devenir de leur création et "lâchent " leur emprise de fabricants-experts selon des modalités plus ou moins réglées. Les " autres" peuvent ainsi devenir les pilotes d'une ouvre laissée disponible à des solutions ouvertes. Ceci suppose de la part de l'artiste, signataire encore de la production, de renoncer à tout ou partie de son travail, de se défaire partiellement d'une maitrise quant à la gestation ou à l'évolution de ce qu'il endosse in fine.

En se plaçant sous la coupe de commanditaires, Nicolas Frespech renoue avec une contrainte qui n'a plus cours dans le champ de l'art actuel. Nous parlons bien ici de commanditaires "à l'ancienne " ordonnant le sujet du travail et non point de commandes, telles qu'une institution ou une collectivité peuvent encore en passer auprès des artistes en choisissant parmi des propositions faites de manière autonome. Dans son Échoppe photographique montée en 2003 avec Bénédicte Albrecht, Nicolas Frespech propose sur le net de réaliser la photographie de votre choix. Quelques lignes où vous décrivez plus ou moins directement ou de manière allusive ce que vous attendez de cette image et la commande est passée. À charge pour lui de passer à l'acte de réalisation,

14. Paul Ardenne, Art, le présent; la création plasticienne au tournant du $\mathrm{xxI}^{\mathrm{e}}$ siècle, Éditions du Regard, 2009. 
dans un délai plus ou moins rapide. Un fichier image de bonne définition est ensuite envoyé gratuitement au commanditaire qui a toute latitude pour l'exploiter à sa guise, l'artiste ne conservant qu'une version basse définition visible sur son site web en regard de la commande originelle.

Se démettre de ses choix ou plutôt se soumettre à ceux des autres relève bien ici d'un renoncement partiel et contraignant dans l'acte de création. Mais cet abandon de maitrise peut donner lieu à deux observations. La première est de rapprocher cet acte de la création artisanale. Au photographe de son quartier, on passe bien commande d'une série de photographies de mariage, de classes maternelles, de familles endimanchées. Le sujet en est fixé par le requérant et le professionnel de l'art n'a plus qu'à faire coïncider son savoir faire avec le désir exprimé. La seconde est de voir une dimension ludique dans ce processus, conférée par la gratuité. Pas question de protester si les délais de livraison s'allongent, si l'image est bien loin des attentes. Il y a plutôt un défi posé à l'artiste pour voir comment il s'en tirera. Comme une histoire commencée par un autre et que l'on poursuit dans un jeu de rôles fictionnel.

La contrainte élective qui consiste à se démettre de la maîtrise complète du processus créatif opère de la même manière un rabattement sur le faire, sur le défi de la fabrication.

Enrôler de nouveaux outils qui a priori ne sont pas faits pour l'art ou se compliquer sciemment la fabrication des images pourrait constituer une autre entrée dans notre inventaire des contraintes électives. Là aussi, quelques exemples, brièvement, peuvent être rassemblés sous cette dénomination sans que la liste en soit close évidemment.

Jeune artiste anglais, Jeremy Wood "dessine" sur son écran grâce à une transmission GPS de ses déplacements réels sur le terrain. Lawn, 2002 est une série de quatre tirages portant chacun le nom des quatre saisons, où l'on peut voir un enchevêtrement de traits plus ou moins denses selon une forme étrange qui se répète d'un panneau à l'autre. Les quatre tracés sont en fait les enregistrements informatiques de la rituelle tonte de pelouse des Anglais - celle de la maison de sa maman en l'occurrence - où l'engin conduit par l'artiste et équipé d'une transmission satellitaire tond et trace des chemins plus ou moins denses selon la poussée saisonnière du gazon! Depuis dix ans, ce jeune artiste démultiplie ses tracés, écrit dans la campagne anglaise en s'équipant lui-même d'un GPS, trace des étoiles malhabiles sur l'Europe en prenant l'avion et en croisant ses destinations, superpose ses chemins aux images de Google Earth. Cette inversion de l'usage d'un outil - savoir où l'on se trouve à tout moment/ fabriquer délibérément une trace enregistrée - est une vraie prouesse technique, complexe et fascinante. Prévu pour nous faire passer de la bidimensionnalité de la carte à la transposition dans le réel de notre orientation, le GPS est ici utilisé exactement en miroir de sa fonction habituelle. Avec Jeremy Wood, la marche 
ou le déplacement sur le territoire produisent une image bidimensionnelle raccordée aux images satellitaires via la transmission des informations. L'outil, avant lui, n'avait jamais servi à générer de l'art; s'en servir de manière exclusive depuis plusieurs années constitue donc une forme de contrainte choisie une fois pour toutes. À charge pour l'artiste d'en inventer les modalités d'exploration, d'en défricher les qualités et de nous régaler d'anecdotes concernant les secrets de fabrication.

Secrets de fabrication également chez ceux qui se compliquent sciemment la création des images: lorsqu'un Philippe Ramette sur Le Balcon défie les lois de la pesanteur ou qu'un Thomas Demand enchaîne les photos de bureaux vides dans une esthétique d'Europe de l'Est des années 1960, on pense d'abord que tout ceci est le produit de logiciels de retouches d'images ou de création 3D. En tout cas amendé par le numérique. Rien de tel lorsque l'on apprend ébahi, que le premier est soutenu dans la réalité par tout un appareillage mécanique sophistiqué destinéà le maintenir à l'équerre du monde et que le second fabrique tous les lieux pris en photo en carton et papier à l'échelle 1! Comme d'autres fabriquent des maquettes de petits trains ou des cathédrales en allumettes.

Dernière proposition pour notre inventaire de la contrainte partielle, la réitération soutenue d'une action qui, isolée, ne serait pas signifiante. Lorsque Julien Prévieux, dépité de ne pas trouver de travail à la sortie d'une formation commerciale, rédige sa première lettre de non-motivation, sait-il que plus d'une centaine suivra, donnant tout son sens à cette rébellion épistolaire jusqu'à la publication de ces courriers ${ }^{15}$ et à l'intégration de sa démarche dans le champ de l'art?

Comme toute pratique d'inventaire, d'archivage, de collecte, la reprise d'un geste quelconque en lui-même peut prendre valeur de démarche artistique lorsqu'il se reproduit et s'étire à l'échelle d'un temps important, lorsqu'il se renouvelle un grand nombre de fois, jouant du même mais non point de l'identique pour reprendre les termes de Michel de M'Uzan' ${ }^{16}$.

\section{Table des matières provisoire}

Il n'est pas sûr du tout que le catalogue ici posé fasse le tour des classifications légitimes et pertinentes qui devraient y figurer. Néanmoins, il propose un éclairage que nous pensons inédit sur quelques exemples de la création contemporaine qui, évidemment, demanderaient à être complétés et approfondis.

Si le sens de l'histoire témoigne d'une forme de libération à l'égard de la contrainte subie, nul ne peut nier qu'elle fait parfois retour sous une forme

15. Julien Prévieux, Lettres de non-motivation, Zones, 2007.

16. Michel de M’Uzan, De l'art à la mort, Éditions Gallimard, 1977. 
choisie, délibérément et sciemment. Cette forme volontariste a souvent pris une ampleur prométhéenne ayant valeur de manifeste, astreinte à des rituels totalisants et contraignants où les artistes, pour citer Queneau, sont comme " des rats qui construisent eux-mêmes le labyrinthe dont ils se proposent de sortir ». Lorsque Georges Perec renonce à la lettre E sur les trois cents pages de La Disparition, il s'impose une discipline restrictive redoutable qui finalement transforme l'objet en un exploit en dehors de toute qualité fictionnelle ou narrative.

Mais loin de limiter notre analyse à quelques figures radicales et souvent citées, l'assujettissement peut fournir un outil d'analyse intéressant y compris lorsque la règle du jeu ne touche qu'une partie de la création. Il y a là comme un processus organique où la contrainte jouerait le rôle de donnée séminale et où la mise en œuvre prendrait tout son sens dans la confrontation avec le réel. Comme une faculté à " jouer » avec le monde, à s'y adapter, à trouver les modalités d'hybridation.

Force est de constater la dimension souvent spectaculaire ou en tout cas fascinante qui se glisse dans de telles pratiques; la "fabrication" s'impose comme le centre d'intérêt majeur de ces créations sous contraintes choisies. Créer de la difficulté là où il pourrait ne pas y en avoir, faire volontairement obstacle, c'est finalement se fabriquer des appuis, des points d'ancrage pour une mécanique captivante.

Au début du XII ${ }^{\mathrm{e}}$ siècle, un ustilz est un équipement, un objet nécessaire que l'on embarque pour un voyage. Le terme donnera deux mots très proches, celui d'outil et celui d'ustensile. De même pouvons-nous penser que les contraintes électives de notre début de $\mathrm{XXI}^{\mathrm{e}}$ siècle sont les ustilz embarqués que d'aucuns choisissent pour leurs voyages. À charge pour eux d'inventer le travail qui va avec. 